PROCEEDINGS OF THE

AMERICAN MATHEMATICAL SOCIETY

Volume 133, Number 12, Pages 3627-3630

S 0002-9939(05)07938-4

Article electronically published on June 3, 2005

\title{
CYCLIC VECTORS IN THE FOCK SPACE OVER THE COMPLEX PLANE
}

\author{
KOU HEI IZUCHI
}

(Communicated by Joseph A. Ball)

\begin{abstract}
In this paper, we characterize the cyclic vectors in the Fock space over the complex plane. We prove that a function $f(z)$ is cyclic in the Fock space if and only if $f(z)$ is a nonvanishing function in $L_{a}^{2}(\mathbb{C})$.
\end{abstract}

\section{INTRODUCTION}

Let $\mathbb{D}$ be the open unit disk of the complex plane $\mathbb{C}$. We denote the polynomial ring on $\mathbb{C}$ by $\mathcal{C}$, and the space of all entire functions by $\operatorname{Hol}(\mathbb{C})$. Let $X$ be a Banach space of holomorphic functions on a domain $\Omega$ in $\mathbb{C}$. For a subset $E$ of $X$, let $\bar{E}$ be the norm closure of $E$ in $X$. In this paper, a function $f$ in $X$ is said to be cyclic if $f \mathcal{C} \subset X$ and $\overline{f \mathcal{C}}=X$. In the classical Hardy space $H^{2}(\mathbb{D})$, it is well known that a function in $H^{2}(\mathbb{D})$ is cyclic if and only if it is $H^{2}(\mathbb{D})$-outer; see $[5$. Also in the Bergman space $L_{a}^{2}(\mathbb{D})$, it is known that $f$ is cyclic if and only if $f$ is $L_{a}^{2}(\mathbb{D})$-outer; see [7.

The Fock space, or the so-called Segal-Bargmann space, is the space of all $\mu$ square-integrable entire functions on $\mathbb{C}$, where

$$
d \mu(z)=\exp \left(-\frac{|z|^{2}}{2}\right) d A(z) / 2 \pi
$$

is the Gaussian measure on $\mathbb{C}$ and $d A$ is the ordinary Lebesgue measure. We denote this space by $L_{a}^{2}(\mathbb{C})=L_{a}^{2}(\mathbb{C}, \mu)$. Then $L_{a}^{2}(\mathbb{C})$ is a Hilbert space with the inner product

$$
\langle f, g\rangle=\frac{1}{2 \pi} \int_{\mathbb{C}} f(z) \overline{g(z)} e^{-\frac{|z|^{2}}{2}} d A(z)
$$

for $f, g \in L_{a}^{2}(\mathbb{C})$, the reproducing kernel functions are given by $K_{\lambda}(z)=e^{\frac{\bar{\lambda} z}{2}}$, $\lambda \in \mathbb{C}$, and the polynomial ring $\mathcal{C}$ is dense in $L_{a}^{2}(\mathbb{C})$; see [2]. We denote by $k_{\lambda}$ the normalized reproducing kernel at $\lambda \in \mathbb{C}$, that is, $k_{\lambda}=\frac{K_{\lambda}}{\left\|K_{\lambda}\right\|}$. It is significant to know which functions in $L_{a}^{2}(\mathbb{C})$ are cyclic. Obviously a cyclic vector $f$ is an entire function without zeros in $\mathbb{C}$, so that we can write $f=e^{h}$ for some $h \in \operatorname{Hol}(\mathbb{C})$. In this paper, we study for which $h \in \operatorname{Hol}(\mathbb{C}), e^{h}$ is cyclic in $L_{a}^{2}(\mathbb{C})$. In the Fock space $L_{a}^{2}(\mathbb{C})$, there is no multiplier of $L_{a}^{2}(\mathbb{C})$ except constant functions; see [6]. So the usual multipication operater $M_{z}$ is not defined on $L_{a}^{2}(\mathbb{C})$. But $M_{z}$ is defined

Received by the editors July 21, 2004 and, in revised form, August 17, 2004.

2000 Mathematics Subject Classification. Primary 46J15, 46H25, 47A16.

Key words and phrases. Fock space, entire function, cyclic vector.

(C)2005 American Mathematical Society 3627

Reverts to public domain 28 years from publication 
on the dense subspace $\mathcal{C}$ of $L_{a}^{2}(\mathbb{C})$. In this meaning, we may say that the definition of the cyclicity in $L_{a}^{2}(\mathbb{C})$ can be considered as cyclic vectors for the densely defined operator $M_{z}$. The following is our main theorem.

Theorem 1.1. Let $h \in \operatorname{Hol}(\mathbb{C})$. Then the following are equivalent:

(i) $e^{h} \in L_{a}^{2}(\mathbb{C})$.

(ii) $h=\alpha z^{2}+\beta z+\gamma$ for $\alpha, \beta, \gamma \in \mathbb{C}$ with $|\alpha|<\frac{1}{4}$.

(iii) $e^{h}$ is cyclic in $L_{a}^{2}(\mathbb{C})$.

It is known that there are nonvanishing functions in $H^{2}(\mathbb{D})$ and $L_{a}^{2}(\mathbb{D})$ which are not cyclic in the respective spaces. But the situation is quite different in the Fock space.

Corollary 1.2. Every nonvanishing function in $L_{a}^{2}(\mathbb{C})$ is cyclic.

\section{Proof OF THE MAIN THEOREM}

The proof of Theorem 1.1 consists of two steps.

Step 1. Let $h \in \operatorname{Hol}(\mathbb{C})$. Then $e^{h} \in L_{a}^{2}(\mathbb{C})$ if and only if $h(z)=\alpha z^{2}+\beta z+\gamma$ with $|\alpha|<\frac{1}{4}$. Moreover if $e^{h} \in L_{a}^{2}(\mathbb{C})$, then $e^{h} \mathcal{C} \subset L_{a}^{2}(\mathbb{C})$.

Proof. Suppose that $e^{h} \in L_{a}^{2}(\mathbb{C})$. First, we shall prove that $h \in \mathcal{C}$ and $\operatorname{deg} h \leq 2$, where $\operatorname{deg} h$ denotes the polynomial degree of $h$. In [3], Chen, Guo, and Hou proved that $\lim _{|\lambda| \rightarrow \infty}\left\langle f, k_{\lambda}\right\rangle=0$ for every $f \in L_{a}^{2}(\mathbb{C})$, so that $\Re h(\lambda)<\frac{|\lambda|^{2}}{4}$ for every $\lambda \in \mathbb{C}$ with sufficiently large modulus. Then obviously $e^{h}$ is an entire function of finite class $p=1$, and the order $\rho$ of $e^{h}$ is

$$
\begin{aligned}
\rho & =\limsup _{r \rightarrow \infty} \frac{\log \left[\max _{|\lambda|=r} \Re h(z)\right]}{\log r} \\
& \leq \limsup _{r \rightarrow \infty} \frac{\log \frac{r^{2}}{4}}{\log r} \\
& =2 .
\end{aligned}
$$

By [1, Corollary 4.5.11], we get that $h \in \mathcal{C}$ and $\operatorname{deg} h \leq 2$.

Now, we show that $e^{\alpha z^{2}+\beta z+\gamma} \notin L_{a}^{2}(\mathbb{C})$ for $\alpha, \beta, \gamma \in \mathbb{C}$ with $|\alpha| \geq \frac{1}{4}$. It is enough to prove that $e^{(a z-b)^{2}} \notin L_{a}^{2}(\mathbb{C})$ for $a, b \in \mathbb{C}$ with $|a| \geq \frac{1}{2}$. Without loss of generality, we may assume that $a \geq \frac{1}{2}$. Write $b=r(\cos \theta+i \sin \theta), r \geq 0$. For $t \in \mathbb{R}$, we have

$$
\left|\left\langle e^{(a z-b)^{2}}, k_{t}\right\rangle\right|=\frac{1}{\left\|K_{t}\right\|}\left|\left\langle e^{(a z-b)^{2}}, K_{t}\right\rangle\right|=\left|e^{b^{2}}\right| e^{\left(a^{2}-\frac{1}{4}\right) t^{2}-2 a t r \cos \theta} .
$$

If $a>\frac{1}{2}$, then we have $\lim _{t \rightarrow \infty}\left|\left\langle e^{(a z-b)^{2}}, k_{t}\right\rangle\right|=\infty$. If $a=\frac{1}{2}$, then we have $\lim _{t \rightarrow \infty}\left|\left\langle e^{(a z-b)^{2}}, k_{t}\right\rangle\right|=\infty$ provided $\cos \theta<0, \lim _{t \rightarrow-\infty}\left|\left\langle e^{(a z-b)^{2}}, k_{t}\right\rangle\right|=\infty$ provided $\cos \theta>0$, and $\left|\left\langle e^{(a z-b)^{2}}, k_{t}\right\rangle\right|=1$ for every $t \in \mathbb{R}$ provided $\cos \theta=0$. Since $\lim _{|\lambda| \rightarrow \infty}\left\langle f, k_{\lambda}\right\rangle=0$ for every $f \in L_{a}^{2}(\mathbb{C})$, we get that $e^{(a z-b)^{2}} \notin L_{a}^{2}(\mathbb{C})$.

Finally, fix $\alpha, \beta, \gamma$ with $|\alpha|<\frac{1}{4}$ and put $h(z)=\alpha z^{2}+\beta z+\gamma$. Let $\epsilon$ be a number satisfying

$$
0<\epsilon<\frac{1}{2}\left(\frac{1}{4}-|\alpha|\right)
$$


For large $|z|,|p(z)| \leq e^{\epsilon|z|^{2}}$ and $|\beta z+\gamma| \leq \epsilon|z|^{2}$. Thus

$$
\begin{aligned}
\left|p(z) e^{h(z)}\right|^{2} & =|p(z)|^{2} e^{2 \Re h(z)} \\
& \leq e^{2 \epsilon|z|^{2}} e^{2(|\alpha|+\epsilon)|z|^{2}} \\
& =e^{2(2 \epsilon+|\alpha|)\left|z^{2}\right|}
\end{aligned}
$$

Then

$$
\left|p(z) e^{h(z)}\right|^{2} e^{-\frac{|z|^{2}}{2}} \leq e^{-\delta|z|^{2}}
$$

for large $|z|$, where $\delta=\frac{1}{2}-2(2 \epsilon+|\alpha|)$. By (1), we have that $\delta>0$. Therefore there exists a positive constant $C$ such that

$$
\int_{\mathbb{C}}\left|p(z) e^{h(z)}\right|^{2} e^{-\frac{|z|^{2}}{2}} d A(z) / 2 \pi \leq C \int_{\mathbb{C}} e^{-\delta|z|^{2}} d A(z) / 2 \pi .
$$

Since the last integral is finite, we get that $e^{h(z)} \mathcal{C} \subset L_{a}^{2}(\mathbb{C})$. This completes the proof.

If $f$ is cyclic, then obviously $f$ is a nonvanishing function. So it remains only to prove that $e^{h(z)}$ is cyclic in $L_{a}^{2}(\mathbb{C})$ for $h(z)=\alpha z^{2}+\beta z+\gamma$ with $|\alpha|<\frac{1}{4}$.

Step 2. If $h(z)=\alpha z^{2}+\beta z+\gamma$ with $|\alpha|<\frac{1}{4}$, then $e^{h(z)}$ is cyclic in $L_{a}^{2}(\mathbb{C})$.

Proof. By Step $1, e^{h(z)} \mathcal{C} \subset L_{a}^{2}(\mathbb{C})$. Let $N$ be a positive integer satisfying

$$
\left(1+\frac{1}{N}\right)|\alpha|<\frac{1}{4} \text {. }
$$

Put $p_{n}(z)=\sum_{k=0}^{n} \frac{\left\{-\frac{1}{N}\left(\alpha z^{2}+\beta z+\gamma\right)\right\}^{k}}{k !}$. Let $l$ be a nonnegative integer. Since

$$
\left|p_{n}(z)\right| \leq \sum_{k=0}^{\infty} \frac{\left\{\frac{1}{N}\left(|\alpha||z|^{2}+|\beta||z|+|\gamma|\right)\right\}^{k}}{k !}=e^{\frac{1}{N}\left(|\alpha||z|^{2}+|\beta||z|+|\gamma|\right)}
$$

we have

$$
\begin{aligned}
& \left|z^{l} p_{n}(z) e^{\alpha z^{2}+\beta z+\gamma}-z^{l} e^{-\frac{1}{N}\left(\alpha z^{2}+\beta z+\gamma\right)} e^{\alpha z^{2}+\beta z+\gamma}\right| \\
& \leq|z|^{l}\left(e^{\left(1+\frac{1}{N}\right)\left(|\alpha||z|^{2}+|\beta||z|+|\gamma|\right)}+e^{\left(1-\frac{1}{N}\right)\left(|\alpha||z|^{2}+|\beta||z|+|\gamma|\right)}\right) \\
& \leq 2|z|^{l} e^{\left(1+\frac{1}{N}\right)\left(|\alpha||z|^{2}+|\beta||z|+|\gamma|\right)}
\end{aligned}
$$

Hence

$$
\begin{aligned}
& \left|z^{l} p_{n}(z) e^{\alpha z^{2}+\beta z+\gamma}-z^{l} e^{-\frac{1}{N}\left(\alpha z^{2}+\beta z+\gamma\right)} e^{\alpha z^{2}+\beta z+\gamma}\right|^{2} e^{-\frac{|z|^{2}}{2}} \\
& \leq 4|z|^{2 l} e^{\left\{2\left(1+\frac{1}{N}\right)|\alpha|-\frac{1}{2}\right\}|z|^{2}+2\left(1+\frac{1}{N}\right)|\beta||z|+2\left(1+\frac{1}{N}\right)|\gamma|}
\end{aligned}
$$

By (2), we have that $2\left(1+\frac{1}{N}\right)|\alpha|-\frac{1}{2}<0$, so that the last function is integrable with respect to $d A$. Since $p_{n}(z) \rightarrow e^{-\frac{1}{N}\left(\alpha z^{2}+\beta z+\gamma\right)}$ pointwise on $\mathbb{C}$ as $n \rightarrow \infty$, by the Lebesgue dominated convergence theorem,

$$
\begin{aligned}
\int_{\mathbb{C}}\left|z^{l} p_{n}(z) e^{\alpha z^{2}+\beta z+\gamma}-z^{l} e^{-\frac{1}{N}\left(\alpha z^{2}+\beta z+\gamma\right)} e^{\alpha z^{2}+\beta z+\gamma}\right|^{2} & e^{-\frac{|z|^{2}}{2}} d A(z) / 2 \pi \\
& \rightarrow 0 \text { as } n \rightarrow \infty
\end{aligned}
$$

Therefore we get

$$
e^{\left(1-\frac{1}{N}\right)\left(\alpha z^{2}+\beta z+\gamma\right)} \mathcal{C} \subset \overline{e^{\alpha z^{2}+\beta z+\gamma} \mathcal{C}} .
$$


Applying this method again for $e^{\left(1-\frac{1}{N}\right)\left(\alpha z^{2}+\beta z+\gamma\right)}$, we have

$$
\begin{array}{r}
\int_{\mathbb{C}}\left|z^{l} p_{n}(z) e^{\left(1-\frac{1}{N}\right)\left(\alpha z^{2}+\beta z+\gamma\right)}-z^{l} e^{-\frac{1}{N}\left(\alpha z^{2}+\beta z+\gamma\right)} e^{\left(1-\frac{1}{N}\right)\left(\alpha z^{2}+\beta z+\gamma\right)}\right|^{2} e^{-\frac{|z|^{2}}{2}} d A(z) \\
\rightarrow 0 \text { as } n \rightarrow \infty
\end{array}
$$

so that

$$
e^{\left(1-\frac{2}{N}\right)\left(\alpha z^{2}+\beta z+\gamma\right)} \mathcal{C} \subset \overline{e^{\left(1-\frac{1}{N}\right)\left(\alpha z^{2}+\beta z+\gamma\right)} \mathcal{C}} .
$$

Continuing this way, we get

$$
\mathcal{C} \subset \overline{e^{\frac{1}{N}\left(\alpha z^{2}+\beta z+\gamma\right)} \mathcal{C}} \subset \cdots \subset \overline{e^{\left(1-\frac{1}{N}\right)\left(\alpha z^{2}+\beta z+\gamma\right)} \mathcal{C}} \subset \overline{e^{\alpha z^{2}+\beta z+\gamma}} .
$$

Thus we get $\overline{e^{\alpha z^{2}+\beta z+\gamma} \mathcal{C}}=L_{a}^{2}(\mathbb{C})$.

\section{ACKNOWLEDGEMENT}

The author thanks the referee for improving the proof.

\section{REFERENCES}

1. C. Berenstein and R. Gay, Complex Variables, Springer-Verlag, New York, 1991. MR.1107514 (92f:30001)

2. X. Chen and K. Guo, Analytic Hilbert Modules, Chapman \& Hall/CRC, 2003. MR.1988884 (2004d:47024)

3. X. Chen, K. Guo, and S. Hou, Analytic Hilbert spaces over the complex plane, J. Math. Anal. Appl., 268 (2002), 684-700. MR.1896222 (2003f:46038)

4. X. Chen and S. Hou, A Beurling-type theorem for the Fock space, Proc. Amer. Math. Soc., 131 (2003), 2791-2795. MR1974336 (2004c:46095)

5. J. Garnett, Bounded Analytic Functions, Academic Press, New York, 1981. MR0628971 (83g:30037)

6. K. Guo and D. Zheng, Invariant subspaces, quasi-invariant subspaces and Hankel operators, J. Funct. Anal., 187 (2001), 308-342. MR1875150 (2003b:47050)

7. H. Hedenmalm, B. Korenblum, and K. Zhu, Theory of Bergman Spaces, Springer-Verlag, New York, 2000. MR1758653 (2001c:46043)

8. K. Hoffman, Banach Spaces of Analytic Functions, Prentice-Hall, Inc., Englewood Cliffs, N.J., 1962. MR0133008 (24:A2844)

9. W. Rudin, Real and Complex Analysis, 3rd ed., McGraw-Hill, 1987. MR0924157 (88k:00002)

Graduate School of Science and Technology, Nitgata University, Nitgata 950-2181, JAPAN

E-mail address: f04n010j@mail.cc.niigata-u.ac.jp 